Check for updates

Cite this: RSC Adv., 2018, 8, 29505

\title{
Application of terpyridyl ligands to tune the optical and electrochemical properties of a conducting polymer
}

\author{
Grzegorz Lisak, (D)*abc Klaudia Wagner, ${ }^{d}$ Jonathan E. Barnsley, ${ }^{e}$ Andrei Veksha, ${ }^{c}$ \\ Gregory Huff, ${ }^{e}$ Anastasia B. S. Elliott, ${ }^{e}$ Pawet Wagner, ${ }^{d}$ Keith C. Gordon, (iDe \\ Johan Bobacka, (D) ${ }^{a}$ Gordon G. Wallace, (D) ${ }^{d}$ Ari Ivaska ${ }^{a}$ and David L. Officer (D) *d
}

\begin{abstract}
We present a simple and effective way of using metal and metal-ligand modifications to tune the electrochemical and optical properties of conducting polymers. To that end, a polyterthiophene functionalized with terpyridine moieties was synthesized and then the resulting film's surface or bulk was modified with different metal ions, namely $\mathrm{Fe}^{2+}, \mathrm{Zn}^{2+}$ and $\mathrm{Cu}^{2+}$ and terpyridine. The modification of the terpyridine functionalized polyterthiophene film by $\mathrm{Fe}^{2+}$ increased the absorptivity and electrochemical capacitance of the conducting polymer, and improved its conjugation. Further modification by $\mathrm{Zn}^{2+}$ and $\mathrm{Cu}^{2+}$ resulted in dramatically different spectroelectrochemical properties of the film. Moreover, the influence of the solvents $\left(\mathrm{ACN}\right.$ and $1: 1 \mathrm{ACN}: \mathrm{H}_{2} \mathrm{O}$ ) in conjunction with the metal ion applied for the modification was found crucial for the electrochemical and optical properties of the films.
\end{abstract}

Received 21st June 2018

Accepted 9th August 2018

DOI: $10.1039 / \mathrm{c} 8 \mathrm{ra05333b}$

rsc.li/rsc-advances

materials, such as molecular wires, dendrimers and hydrogels as well as polymers with terpyridine moieties in the polymer backbone or in the side chains. ${ }^{13-19}$ Additionally, the functionalization of conducting polymers by adding the terpyridine moiety as a side-group has led to the development of chemical sensors and tunable surfaces via molecular engineering and surface modifications of the metallo-organic complexes at the interface. ${ }^{20-22}$

The challenge in adding bulky substituents such as terpyridines to polythiophenes using functionalized thiophene monomers is the distortion in the polymer backbone that arises from neighboring substituent steric interactions. In order to overcome this, Officer and co-workers have utilized functionalized terthiophenes, although the polymerization of terthiophenes can be challenging particularly with conjugated substituents. ${ }^{23-25}$ The successful formation of conducting polymers from terthiophenes has however been achieved by the attachment of alkoxy substituents on the outer rings of the thiophene monomers, that not only activates the outer thiophene rings for oxidative coupling but also reduces charge localization within an aryl-aryl conjugated system that deactivates terthiophene monomer oxidative coupling. ${ }^{20,26,27}$

In this work, a bisdecyloxy-substituted terthiophene

aJohan Gadolin Process Chemistry Centre, Laboratory of Analytical Chemistry, Åbo Akademi University, FIN-20500 Åbo-Turku, Finland

${ }^{b}$ School of Civil and Environmental Engineering, Nanyang Technological University, 50 Nanyang Avenue, Singapore 639798, Singapore

'Nanyang Environment and Water Research Institute, 1 Cleantech Loop, CleanTech, Singapore 637141, Singapore

${ }^{d} A R C$ Centre of Excellence for Electromaterials Science, Intelligent Polymer Research Institute, University of Wollongong, NSW 2522 Wollongong, Australia

${ }^{e}$ Chemistry Department, University of Otago, Dunedin, New Zealand monomer was functionalized with a vinylterpyridine substit-

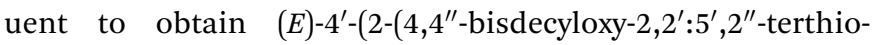
phen-3'-yl)ethenyl)-2, $2^{\prime}: 6^{\prime}, 2^{\prime \prime}$-terpyridine (TTPy), which was subsequently modified with metal ions (Fig. 1A). Modification of the film surface and the bulk of poly(TTPy) with $\mathrm{Fe}^{2+}, \mathrm{Zn}^{2+}$ and $\mathrm{Cu}^{2+}$ metal ions afforded dramatically different optical and electrochemical film properties, with the $\mathrm{Fe}^{2+}$-modified 
A)

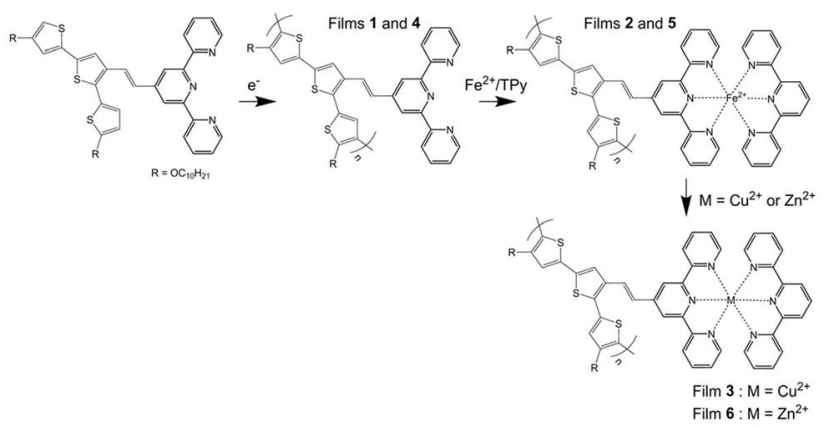

B)

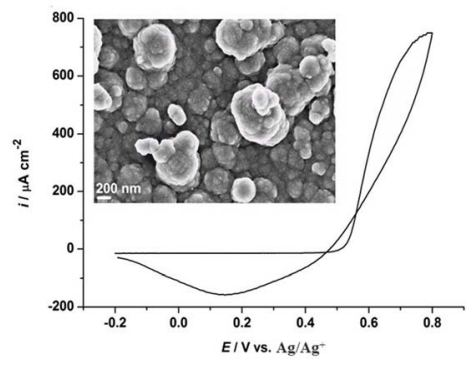

C)

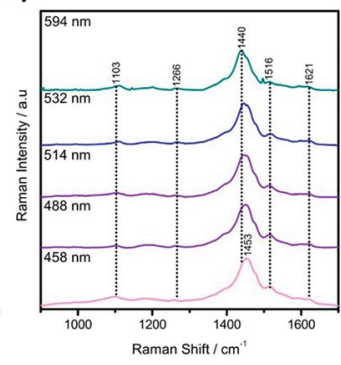

Fig. 1 (A) Structural diagram of the preparation of poly(TTPy) films 16. (B) Electropolymerisation of poly(TTPy) on ITO substrate (area 1.55 $\mathrm{cm}^{2}$ ) performed by cycling the potential, during a single full cycle, between -0.2 and $0.8 \mathrm{~V}$ (vs. $\mathrm{Ag} / \mathrm{AgCl} / 0.1 \mathrm{M}$ TBAP-ACN reference electrode) with a scan rate of $0.1 \mathrm{~V} \mathrm{~s}^{-1}$ in the solution containing $5 \mathrm{mM}$ TTPy monomer and 0.1 M TBAP in $1: 1$, ACN : DCM. Inset: SEM picture of a poly(TTPy) film obtained using the same procedure for electropolymerisation but applied after 5 potential cycles. (C) Resonance Raman data for a poly(TTPy) film.

film showing an increased electrochemical capacitance, better conjugation of the conducting polymer system and higher absorptivity.

\section{Experimental}

\section{Electrosynthesis of poly(TTPy) and its surface modifications by metal-TPy}

Cyclic voltammetry was used to electrosynthesize poly(TTPy) films on indium tin oxide (ITO) glass electrodes. The electropolymerisation was done using eDAQ system controlled by EChem software in a three electrodes system in $1: 1 \mathrm{ACN}$ : DCM solution containing $5 \mathrm{mM}$ TTPy monomer with $0.1 \mathrm{M}$ TBAP as supporting electrolyte. To avoid solubility problems, it was important to first dissolve the monomer in DCM while the supporting electrolyte salt (TBAP) was dissolved in ACN, followed by mixing of the two solutions to form the solution for polymerization. Before the polymerization, the ITO slide (area $1.55 \mathrm{~cm}^{2}$ ) was repeatedly washed with deionized water and ethanol ( 5 times) and left in ethanol for 10 min under ultrasound conditions, after which it was dried in open air. Such a pretreated ITO slide served as the working electrode. A platinum mesh electrode served as the counter electrode, while a $\mathrm{Ag} / \mathrm{Ag}^{+} / 0.1 \mathrm{M}$ TBAP in ACN reference electrode was used. Prior to the electrosynthesis, the reference electrode was calibrated in ferrocene solution. The electrosynthesis was done by

performing one potential cycle between -0.2 and $0.8 \mathrm{~V}(v \mathrm{~s}$. Ag/ $\mathrm{Ag}^{+} \mathrm{l} / 0.1 \mathrm{M}$ TBAP-ACN electrode) with the scan rate of $0.1 \mathrm{~V} \mathrm{~s}^{-1}$. After the polymerization the poly(TTPy) film on ITO glass was washed with ACN (films 1 and 4).

The metal complexation of the poly(TTPy) was done by first soaking the poly(TTPy) films on ITO glass for $1 \mathrm{~h}$ in ACN solution containing $10^{-3} \mathrm{M} \mathrm{FeCl}_{2}$. This was done in order to immobilize $\mathrm{Fe}^{2+}$ in mono complexes with terpyridyl moieties present in the poly(TTPy). Then the addition of $5 \times 10^{-3} \mathrm{M}$ TPy was done to cap the terpyridyl-Fe ${ }^{2+}$ mono complex with another terpyridyl group $\left(\mathrm{Fe}^{2+}\right.$-based modifications, films 2 and 5). The films were left in the $10^{-3} \mathrm{M} \mathrm{FeCl}_{2}$ and $5 \times 10^{-3} \mathrm{M}$ TPy in ACN solution for $24 \mathrm{~h}$. Then the films were washed with ACN and characterized by using cyclic voltammetry, optical spectroscopy, X-ray photoelectron spectroscopy (XPS) and contact angle measurement done using a goniometer. After that, the $\mathrm{Fe}^{2+}$ containing films were further used for subsequent modification by $\mathrm{Cu}^{2+}$ and $\mathrm{Zn}^{2+}$. The exchange of the central metal ion (from $\mathrm{Fe}^{2+}$ to $\mathrm{Cu}^{2+}$ or $\mathrm{Zn}^{2+}$ ) was done by soaking the films for $24 \mathrm{~h}$ either in the ACN solution containing $10^{-3} \mathrm{M} \mathrm{CuCl}_{2}$ (poly(TTPy) $\mathrm{Cu}$ film 3) or in the $1: 1 \mathrm{ACN}: \mathrm{H}_{2} \mathrm{O}$ solution containing $10^{-3} \mathrm{M}$ $\mathrm{ZnCl}_{2}$ (poly(TTPy)Zn film 6). Again, after the modification, the films were washed with ACN and characterized by using cyclic voltammetry, optical spectroscopy, XPS and contact angle measurement.

\section{Characterization of poly(TTPy), poly(TTPy)Fe, poly(TTPy)Cu and poly(TTPy)Zn}

The thickness of the obtained poly(TTPy) film on ITO was investigated using profilometry (Dektak 150 profilometer). Field emission gun scanning electron microscopy (FEG-SEM, Leo 1530, Zeiss) was used in order to investigate the morphology of poly(TTPy). For that purpose, a thicker film was electrosynthesized as described above, but by applying five potential cycles. Resonance Raman measurements were done on the poly(TTPy) electrodeposited on ITO glass in order to confirm the conducting polymer structure. Spectra were collected using a setup which has been previously described. ${ }^{28}$ In short, it is composed of an excitation beam and collection lens in a $135^{\circ}$ backscattering arrangement. Scattered photons were focused on the entrance slit of an Acton SpectraPro500i spectrograph with a 1200 grooves per $\mathrm{mm}$ grating, which disperses the radiation in a horizontal plane on a Princeton Instruments Spec10 liquid-nitrogen-cooled CCD detector. A Coherent Innova Sabre argon-ion laser provided 457.9, 488.0 and $514.5 \mathrm{~nm}$ excitation wavelengths, while solid-state CrystaLasers were used for 532.0 and $593.7 \mathrm{~nm}$. Notch filters (Kaiser Optical, Inc.) or long-pass filters (Semrock, Inc.) matched to these wavelengths was used to remove the laser excitation line, and a $632.8 \mathrm{~nm}$ short pass filter was used to remove a sharp peak from the $593.7 \mathrm{~nm}$ line.

Cyclic voltammetry was performed by recording voltammograms in $0.1 \mathrm{M}$ TBAP in ACN (for poly(TTPy)Cu) or in $1: 1$ ACN : $\mathrm{H}_{2} \mathrm{O}$ (poly(TTPy)Zn) by scanning the potential (five scans, only fifth scan shown) between 0.0 and $1.0 \mathrm{~V}\left(v s . \mathrm{Ag} / \mathrm{Ag}^{+} / 0.1 \mathrm{M}\right.$ TBAP-ACN reference electrode) with the scan rate of $0.1 \mathrm{~V} \mathrm{~s}^{-1}$. Optical spectroscopy was done on a dry film using a Shimadzu 
UV-1800 spectrophotometer (Kyoto, Japan) by recording the absorbance spectra of a film in air from 400 to $800 \mathrm{~nm}$. The spectra obtained for pure ITO glass was used for background correction. For the XPS analysis the samples were cut into approximately $7 \times 7 \mathrm{~mm}^{2}$ squares and mounted on a copper sample holder using carbon tape. The samples were grounded using silver paste connecting the ITO surface to the copper sample holder. XPS spectra were collected by illuminating the samples with a dual anode, non-monochromatic X-ray source (Omnivac) using $\mathrm{Mg} \mathrm{K} \alpha$ radiation and the photoemission collected by a SES2002 analyzer (Scienta) operating at $200 \mathrm{eV}$ (survey scan) or $50 \mathrm{eV}$ (detail scan) pass energy. The spectra were processed using CasaXPS. A linear background was used, and curve fitting was carried out by restricting the width of the fitted peaks to a maximum of the resolution expected for the analyzer configuration ( $4 \mathrm{~mm}$ slit, $50 \mathrm{eV}$ pass energy has a theoretical FWHM of $1 \mathrm{eV}$ ). The work function of the analyzer was approximately $6 \mathrm{eV}$. This and any charging shifts were accounted for by referencing the energy scale to the carbon peak at $284 \mathrm{eV}$. Quantitative results were obtained from the survey scans using the relative sensitivity factors in the CasaXPS library. No correction for analyzer transmission as a function of electron energy has been applied, which will tend to reduce the apparent concentration of the high binding energy elements by $30-40 \%$. Finally, the hydrophobicity of all surfaces was characterized by Data-Physics OCA20 goniometer and water droplet ( $2 \mu \mathrm{L}$, Milli Q water). The contact angle was measured just after the water droplet was in contact with the measured surface. The uncertainty of the measurements was always obtained from five consecutive measurements.

\section{The $\mathrm{Fe}\left[(\mathrm{TTPy})_{2}\right]^{2+}$ complex formation with competing ions}

The Fe $\left[(\text { TTPy })_{2}\right]^{2+}$ complex formation was investigated in presence of competing ions utilizing membrane entrapped TPPy and immobilized onto paper strips. To prepare such substrate a paper strip was dip coated in a THF solution of $1 \%$ TTPy, $0.35 \%$ KTFPB, $65.65 \% o$-NPOE and 33\% PVC (weight\%) and left for evaporation of the solvent. Entrapment of the monomer is such matrix allowed to follow kinetically the formation of $\mathrm{Fe}$ $\left[(\mathrm{TTPy})_{2}\right]^{2+}$ complex in presence of competing ions. The measurements were performed using two different protocols. In the first protocol the measurement was done by measuring absorbance of $\mathrm{Fe}\left[(\mathrm{TTPy})_{2}\right]^{2+}$ complex formation at $598 \mathrm{~nm}$ for $300 \mathrm{~s}$ at $0.1 \mathrm{~mol} \mathrm{dm}^{-3} \mathrm{FeCl}_{2}$. Then the measurements were done when $\mathrm{Fe}^{2+}$ was competing for complex formation with a $1: 1$ ratio of competing ions ( $\mathrm{j}$ ) to obtain the response of the $\mathrm{Fe}$ $\left[(\mathrm{TTPy})_{2}\right]^{2+}$ affected complex formation. The sequence in which the interfering ions were investigated was as follow: $\mathrm{Na}^{+}, \mathrm{K}^{+}$, $\mathrm{Mg}^{2+}, \mathrm{Ag}^{+}, \mathrm{Ca}^{2+}, \mathrm{Al}^{3+}, \mathrm{Sr}^{2+}, \mathrm{Zn}^{2+}, \mathrm{Fe}^{3+}, \mathrm{NH}^{4+}, \mathrm{Ba}^{2+}, \mathrm{Mn}^{2+}, \mathrm{Hg}^{2+}$, $\mathrm{Ni}^{2+}, \mathrm{Co}^{2+}, \mathrm{Cu}^{2+}$ and $\mathrm{Ru}^{3+}$. Each competing ion was investigated using a new $\mathrm{Fe}^{2+}$-SM paper strip. The influence of the competing ion on $\mathrm{Fe}^{2+}$ complexation by TTPy was determined then as the ratio between the maximum complex absorbances at $598 \mathrm{~nm}$ after $300 \mathrm{~s}$ of the competing ion-free and competing ion responses. In the second protocol, the absorbance spectra were taken in air from dried (approx. $1 \mathrm{~h}$ ) paper strips used in the first protocol. The absorbance measurements were performed between 400 and $700 \mathrm{~nm}$. The influence of the interfering ion on $\mathrm{Fe}^{2+}$ complexation by TTPy was then determined as the ratio between the $\mathrm{Fe}\left[(\mathrm{TTPy})_{2}\right]^{2+}$ complex absorbance at $598 \mathrm{~nm}$ of competing ion-free and competing ion responses. A relatively high noise level in the absorption spectra between 450 to approx. $550 \mathrm{~nm}$, attributed to the presence of paper in the measuring cell, was always observed when investigating paperbased strips.

\section{Computational modelling}

In order to validate the obtained poly(TTPy) and its modification, geometry and vibrational calculations were generated using the Gaussian $09 \mathrm{~W}$ program package which implemented the Becke, three-parameter, Lee-Yang-Parr (B3LYP) functional employing a LANL2DZ basis set for Fe centers and 6-31G(d) for all other atoms. ${ }^{29}$ No negative frequencies were observed for these optimized geometries, which indicated that local minima had been achieved. $\mathrm{Fe}^{2+}$ was assumed to be low spin with a singlet spin state. $\mathrm{Cu}^{2+}$ and $\mathrm{Zn}^{2+}$ were calculated with doublet and singlet spin states respectively. Formation energies $(\Delta G)$ were calculated for each metal complex using a simplified terthiophene unit to mimic the energetics of the larger polythiophene, while avoiding a high computational demand. While larger polymer units may have changed the overall energetics, the relative binding preferences were constrained by the local electronic and geometric nature of the terpyridine residue. Relative formation energies $(\Delta G)$ for each complex were calculated using a products-reactants system as shown in eqn (1)-(3):

$$
\begin{aligned}
\mathrm{Fe}(\mathrm{MeCN})_{6}{ }^{2+}+\mathrm{TTPy}+\mathrm{TPy} \rightarrow & \mathrm{Fe}[\mathrm{TTPyTPy}]^{2+}+6 \mathrm{MeCN} \\
& (\text { for terpy binding) } \\
\mathrm{Cu}(\mathrm{MeCN})_{4}{ }^{2+}+\mathrm{TTPy}+\mathrm{TPy} \rightarrow & \mathrm{Cu}[\mathrm{TTPyTPy}]^{2+}+4 \mathrm{MeCN} \\
& (\text { for terpy binding) }
\end{aligned}
$$

$\mathrm{Zn}(\mathrm{MeCN})_{4}{ }^{2+}+$ TTPy + TPy $\rightarrow \mathrm{Zn}[\mathrm{TTPyTPy}]^{2+}+4 \mathrm{MeCN}$ (for terpy binding)

Optimized energies were used for all substituents and these structures yielded no negative frequencies as above.

\section{Results and discussion}

\section{Electrosynthesis and characterization of poly(TTPy)}

The TTPy has been previously synthesized and used in the development of paper- and glass-based disposable iron(II) optical sensors. ${ }^{4}$ In this sensor design, two properties of the monomer led to the development of novel time- and concentration-based analytical methods through, (i) the ability to complex metal ions by the terpyridyl moiety and (ii) deceleration of the complex formation by the presence of the terthiophene moiety. The $\mathrm{Fe}\left[(\mathrm{TTPy})_{2}\right]^{2+}$ complex has an absorbance maximum at $591 \mathrm{~nm}$, which is not masked by the absorption of TTPy complexes with any other metal ions (spectroscopic fingerprint of metal-terpyridine complex). ${ }^{4}$ The 
polymerization of TTPy, however, offered the opportunity to further expand the sensing capabilities of these species.

Conducting polymer films of poly(TTPy) ( 1 and 4) could be obtained by electropolymerization of the TTPy terthiophene moiety (Fig. 1A). The electrodeposition of poly(TTPy) by cyclic voltammetry is shown in Fig. 1B. A thin conducting polymeric film was obtained during a full single potential cycle. When scanning the potential from -0.2 to $0.8 \mathrm{~V}$ using a scan rate of $0.1 \mathrm{~V} \mathrm{~s}^{-1}$, the polymer film formation began at $0.55 \mathrm{~V}$ as a sharp increase of the current density, followed by an oxidation peak maximum around $0.8 \mathrm{~V}$.

On the reverse scan from 0.8 to $-0.2 \mathrm{~V}$, a reduction peak was recorded at $0.15 \mathrm{~V}$ corresponding to the reduction of the electrodeposited poly(TTPy). Such a polymerization pattern is typical of terthiophene-based polymers, ${ }^{30,31}$ consequently, single $\mathrm{CV}$ cycles up $0.8 \mathrm{~V}$ were used to grow the thin polymer films (the measured thickness was approx. $95 \mathrm{~nm}$ ). For thicker films that could be used for SEM analysis, electropolymerisation was undertaken over five consecutive potential cycles. The morphology of the film is shown in the inset of Fig. 1B. The SEM image showed full ITO coverage by the poly(TTPy) with well pronounced globular features.

Resonance Raman measurements were performed to characterize the poly(TTPy) film and are presented in Fig. 1C. The data showed a number of weak spectral features, which are dominated by the band at $1440-1453 \mathrm{~cm}^{-1}$. This band is well known as the thiophene line B and is visualized in Fig. 2A. The comparative strength of this band indicates that thiophene is involved in the transition occurring for these wavelengths. The next strongest bands at 1103 and $1516 \mathrm{~cm}^{-1}$, are predicted to be terthiophene based (Fig. 2A). This is consistent with electronic transitions which are terthiophene localised. Characteristic terpyridyl signals (typically around 1000, 1360, 1560 and $1590 \mathrm{~cm}^{-1}$ ) are not observed. ${ }^{32}$

TD-DFT data indicated this transition is of HOMO to LUMO nature, and elicits the transfer of electron density from a terthiophene orbital to an orbital delocalized over the terthiophene, $\mathrm{C}=\mathrm{C}$ and terpy units (Fig. 2B). The $\mathrm{B}$ line vibration exhibits 'frequency dispersion', a redshift of band frequency as the excitation wavelength is increased, as a result of a high

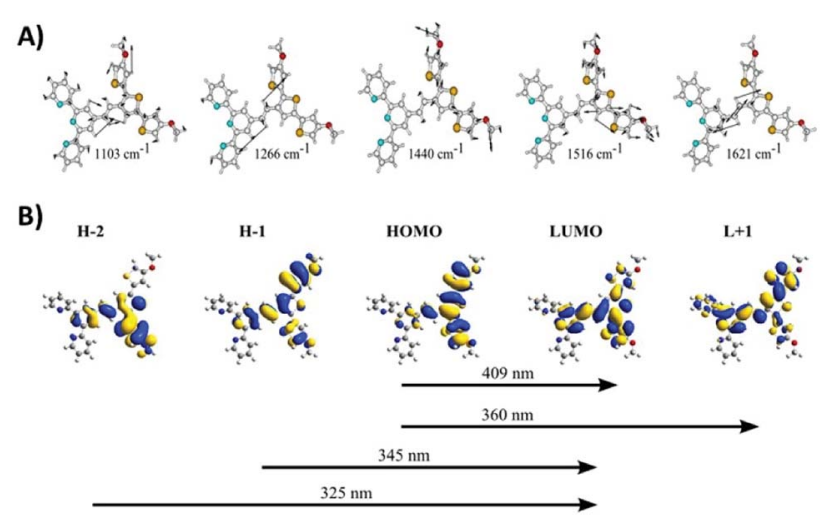

Fig. 2 (A) DFT generated vibrational eigenvector diagrams for TPPy. (B) TD-DFT generated orbitals for a TPPy unit calculated at B3LYP/6$31 \mathrm{G}(\mathrm{d})$ level of theory. polarizability. ${ }^{33}$ This effect is uncommon in thiophene oligomers/polymers due to an increasing presence of $\pi$-stacking as the thiophene backbone is elongated; $\pi$-stacking results in a partial cancelation of $\pi$-delocalisation, despite an increased thiophene length. A 'soft' B line, which disperses as a function of excitation wavelength, suggests that $\pi$-stacking in this polymer film is minimal. ${ }^{34} \mathrm{Such}$ a result is expected due to the steric bulk of the terpyridine residues, which may make $\pi-\pi$ interactions between thiophene units difficult.

\section{Modification of poly(TTPy) with $\mathrm{Fe}^{2+}$}

The electropolymerised poly(TTPy) films on ITO glass (1 and 4) were treated with $\mathrm{Fe}^{2+}$ followed by TPy to give poly(TTPy)Fe films (2 and 5) (Fig. 1A). Two films were prepared in order to allow further modification with $\mathrm{Cu}^{2+}$ and $\mathrm{Zn}^{2+}$ ions. The electrochemical and optical properties of poly(TTPy) before and after $\mathrm{Fe}^{2+}$ modification are shown in Fig. 3A and $\mathrm{B}$ (films 1 and 2) and Fig. 3C and D (films 4 and 5).

From this electrochemical and optical study, it can be seen that the poly(TTPy) films 1 has a broad oxidation peak with the oxidation maximum around $0.75 \mathrm{~V}$ and film absorption maximum at $550 \mathrm{~nm}$. The modification of poly(TTPy) film 1 by $\mathrm{Fe}^{2+} /$ TPy (film 2) dramatically influenced its properties. The oxidation peak of the modified film was shifted towards lower potential values, namely $0.5 \mathrm{~V}$, and resulted in higher electrochemical capacitance. This phenomenon indicated that the conjugation of the poly(TTPy) was improved due to the introduction of $\mathrm{Fe}^{2+} / \mathrm{TPy}$ to the film. This conjugation improvement may also be confirmed by the bathochromic shift in the absorbance maximum of film 2 (Fig. 3B) although, given the
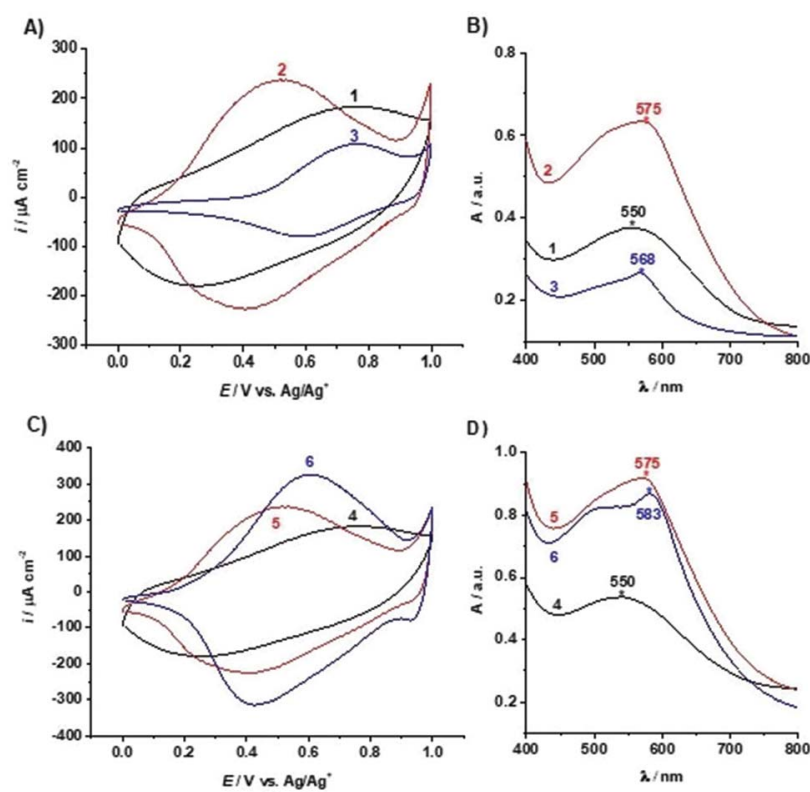

Fig. 3 Cyclic voltammograms (fifth scan shown) performed by cycling the potential between 0.0 and $1.0 \mathrm{~V}$ (vs. Ag/ $/ \mathrm{Ag}^{+} / 0.1 \mathrm{M}$ TBAP-ACN reference electrode) with the scan rate of $0.1 \mathrm{~V} \mathrm{~s}^{-1}$ in the solution containing 0.1 M TBAP in ACN of films: (A) 1-3 and (C) 4-6. (B) UV-VIS spectra of films in air between 400 and $800 \mathrm{~nm}$ of films: (C) 1-3 and (D) 4-6. 
marked increase in absorption intensity, this must also be contributed to by the Fe[TTPy(TPy) $]^{2+}$ complex. The metal-toligand charge-transfer (MLCT) band absorption for $\mathrm{Fe}$ $\left[(\mathrm{TPy})_{2}\right]^{2+}$ occurs in solution at $552 \mathrm{~nm}$ while that of Fe(TTPy $)_{2}$ is substantially red shifted to $591 \mathrm{~nm}^{4}{ }^{4}$ Therefore it is not surprising that the asymmetric Fe[TTPy(TPy) $]^{2+}$ complex would not be so red-shifted and absorb around $575 \mathrm{~nm}$. Thus, it would appear that during the film modification with $\mathrm{Fe}^{2+} / \mathrm{TPy}$, not only is the surface of the film modified but also the bulk of the conducting polymer. In addition, the molar extinction coefficient (absorptivity) of the film visibly changed after modification by $\mathrm{Fe}^{2+} /$ TPy. This increase is likely due to both the increase in polymer conjugation as well as incorporation of $\mathrm{Fe}^{2+} / \mathrm{TPy}$ into the polymer resulting in complex formation.

XPS and contact angle measurement of the surface of films before and after modifications were performed and the results are given in Fig. 4 and Table 1. The XPS analysis confirmed the presence of iron in the modified film (Fig. 4A). The contact angles before and after modification by $\mathrm{Fe}^{2+}$ and $\mathrm{Fe}^{2+}$-TPy did not differ.

\section{Stability of the $\mathrm{Fe}\left[(\mathrm{TTPy})_{2}\right]^{2+}$ complex in the presence of competing ions}

Owing to the strong affinity of the terpyridine moiety towards various metal ions, the poly(TTPy)Fe film could be further modified. To this end, we undertook an additional study to select the most suitable metals ions to replace $\mathrm{Fe}^{2+}$ in the polymer film. In order to avoid the overlap of the broad absorption of the poly(TTPy) at $550 \mathrm{~nm}$ (Fig. 3B) with that of the absorption due to the polymer TTPy/Fe complex at $598 \mathrm{~nm}$ (Fig. 5B), we used monomer (TTPy) to investigate the formation of the $\mathrm{Fe}\left[(\mathrm{TTPy})_{2}\right]^{2+}$ complex in the presence of seventeen different cations $\left(\mathrm{Na}^{+}, \mathrm{K}^{+}, \mathrm{Mg}^{2+}, \mathrm{Ag}^{+}, \mathrm{Ca}^{2+}, \mathrm{Al}^{3+}, \mathrm{Sr}^{2+}, \mathrm{Zn}^{2+}, \mathrm{Fe}^{3+}\right.$, $\mathrm{NH}_{4}^{+}, \mathrm{Ba}^{2+}, \mathrm{Mn}^{2+}, \mathrm{Hg}^{2+}, \mathrm{Ni}^{2+}, \mathrm{Co}^{2+}, \mathrm{Cu}^{2+}$ and $\left.\mathrm{Ru}^{3+}\right)$ with the kinetic procedures (protocols) developed in our previous study ${ }^{4}$ (Fig. 5). The TTPy monomer was incorporated into a polymer

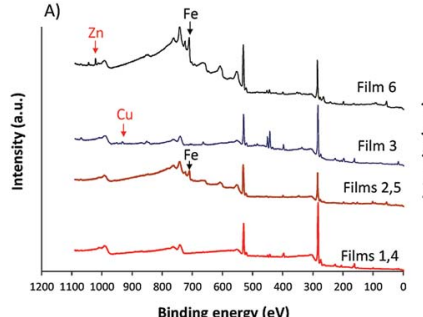
Binding energy (eV)
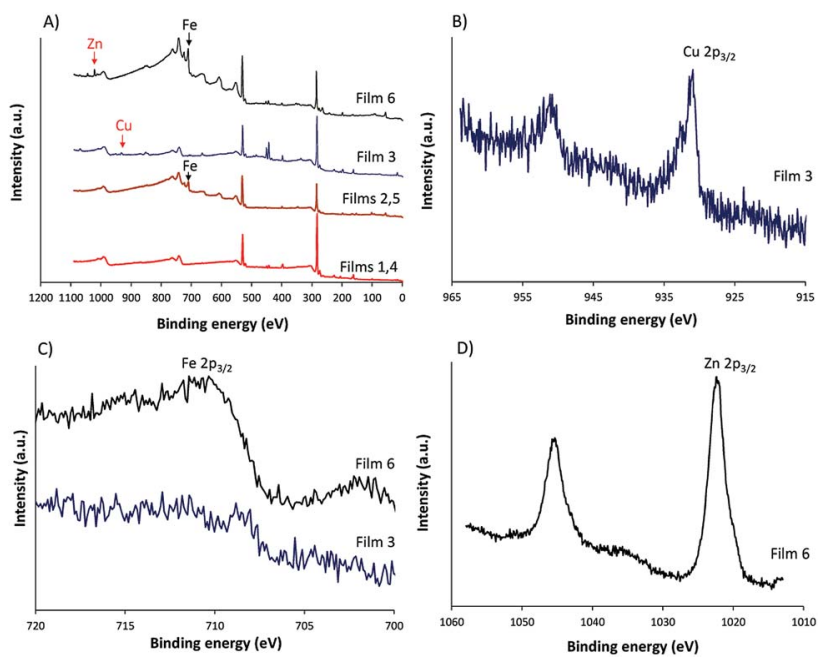

Fig. 4 (A) XPS survey spectra of all films, (B) Cu $2 p$ core level spectra of film 3, (C) Fe $2 p$ core level spectra of films 3 and 6 and (D) Zn 2p core level spectra of film 6 .
Table 1 Elemental composition and contact angle data for poly(TTPy), poly(TTPy)Fe, poly(TTPy)Cu and poly(TTPy)Zn films

\begin{tabular}{lllllllll}
\hline No. & $\mathrm{C} \%$ & $\mathrm{~N} \%$ & $\mathrm{O} \%$ & $\mathrm{~S} \%$ & $\mathrm{Fe} \%$ & $\mathrm{Cu} \%$ & $\mathrm{Zn} \%$ & $\theta_{\mathrm{c}}$ degrees \\
\hline $\mathbf{1 , 4}$ & 77.44 & 3.5 & 14.9 & 4.1 & $\mathrm{~N}$ & - & - & $104 \pm 3.2$ \\
$\mathbf{2 , 5}$ & 68.9 & 1.6 & 26.0 & 1.9 & 1.6 & - & - & $99.7 \pm 2.6$ \\
$\mathbf{3}$ & 75.8 & 3.8 & 16.6 & 3.6 & - & 0.2 & - & $88.8 \pm 1.8$ \\
$\mathbf{6}$ & 63.7 & 1.8 & 29.0 & 1.7 & 3.5 & - & 0.3 & $85.5 \pm 10.5$
\end{tabular}

membrane on paper as to mimic the solid environment of the polymer. As shown by both measurement protocols (see Experimental section and ref. 4), the $\mathrm{Cu}^{2+}$ and $\mathrm{Zn}^{2+}$ ions yielded the lowest absorbances of TTPy/Fe complex formation, indicating that these two metal ions were competing for complex formation with $\mathrm{Fe}^{2+}$, this suggested that $\mathrm{Cu}^{2+}$ and $\mathrm{Zn}^{2+}$ ions could be a good candidates for decomplexing Fe from the pol$y$ (TTPy)Fe films.

\section{Modification of poly(TTPy) with $\mathrm{Cu}^{2+}$ and $\mathrm{Zn}^{2+}$}

The poly(TTPy)Fe was further modified by $\mathrm{Cu}^{2+}$ and $\mathrm{Zn}^{2+}$ to obtain poly(TTPy)Cu and poly(TTPy)Zn films with different spectroelectrochemical properties. The electrochemical and optical properties of poly(TTPy)Cu (film 3) shown in Fig. 3A and B and poly(TTPy)Zn (film 6) in Fig. 3C and D. The oxidation potential of poly(TTPy)Cu was similar to that of unmodified poly(TTPy) $(0.75 \mathrm{~V})$ while the oxidation potential of poly(TTPy) $\mathrm{Zn}$ was $0.6 \mathrm{~V}$. In both cases, loss in conjugation of the polymeric film would account for the shift in the oxidation potential towards higher potentials. This decrease in polymer conjugation was also observed in UV-VIS spectra, where the absorption spectra from poly(TTPy) decreased. The absorbance peak maximum for the poly(TTPy)Cu was $568 \mathrm{~nm}, 7 \mathrm{~nm}$ less than that of poly(TTPy)Fe. For the film modified by $\mathrm{Zn}^{2+}$ and $\mathrm{Zn}^{2+}$-TPy, the absorbance peak maximum for the new material was recorded at $583 \mathrm{~nm}, 8 \mathrm{~nm}$ more than that of poly(TTPy)Fe. Both modifications resulted in more hydrophilic films compared to pure poly(TTPy) and poly(TTPy)Fe as evidenced by the contact angle data in Table 1. Moreover, XPS analysis (Table 1) revealed that for the poly(TTPy)Cu film, all the $\mathrm{Fe}^{2+}$ was exchanged by $\mathrm{Cu}^{2+}$, while in the case of poly(TTPy)Zn, only a partial exchange, at
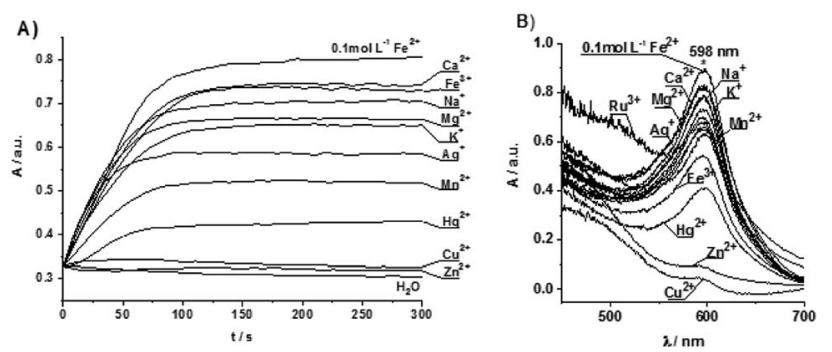

Fig. 5 (A) Competing ion measurements performed by recording the absorbance at a single wavelength $(598 \mathrm{~nm})$ for Fe[(TTPy $\left.)_{2}\right]^{2+}$ complex formation utilizing TPPy-SM deposited on the paper substrates in the solution containing $0.1 \mathrm{~mol} \mathrm{dm}^{-3}$ of $\mathrm{Fe}^{2+}$ or $0.1 \mathrm{~mol} \mathrm{dm}^{-3}$ of $\mathrm{Fe}^{2+}$ and $0.1 \mathrm{~mol} \mathrm{dm}^{-3}$ of interfering ions (1:1): during $300 \mathrm{~s}$ measurement time. (B) Post measurement absorbance, recorded between 400 and $700 \mathrm{~nm}$, performed in air on the dry paper strips. 
least at the surface was observed. The electrochemical capacitance of the poly(TTPy)Zn film was similar to poly(TTPy)Fe. However, the electrochemical capacitance of poly(TTPy)Cu decreased dramatically compared to that of poly(TTPy)Zn or poly(TTPy)Fe, providing the information that introduced $\mathrm{Cu}^{2+}$ influenced the integrity of the modified film, thus reducing its electroactivity. This fact indicated the loss in the conjugation of the modified film. The poly(TTPy)Zn film remain the integrity of the poly(TTPy) as the peak maximum of the conducting polymer, around $550 \mathrm{~nm}$ remain. The major difference between poly(TTPy)Cu and poly(TTPy)Zn was the solvent used, where for $\mathrm{Zn}^{2+}$ and $\mathrm{Cu}^{2+}$ modifications $1: 1 \mathrm{ACN}: \mathrm{H}_{2} \mathrm{O}$ and ACN were applied, respectively. In the presence of ACN partial release of $\mathrm{Cu}^{2+}$ may occur that further on acts as oxidizing agent for the conducting polymer film. This phenomenon was investigated and reported in $\mathrm{Cu}^{2+} / \mathrm{ACN}$ polythiophene aggregates chemistry. ${ }^{35}$ Moreover, the XPS core level spectra (Fig. 4B) revealed that the binding energy of $\mathrm{Cu} 2 \mathrm{p}_{3 / 2}$ in poly(TTPy)Cu was $\sim 932$, which corresponds to $\mathrm{Cu}(\mathrm{I})$ and/or $\mathrm{Cu}(0) .{ }^{36,37}$ These data indicated that redox reactions may have influenced on the stability of the system and caused reduction of $\mathrm{Cu}^{2+}$ on the surface of film. The binding energy of $\mathrm{Zn} 2 \mathrm{p}_{3 / 2}$ in poly(TTPy)Zn was $\sim 1022 \mathrm{eV}$ (Fig. 4D), which is attributed to $\mathrm{Zn}$ (II) species. ${ }^{38}$ The binding energy of $\mathrm{Fe} 2 \mathrm{p}_{3 / 2}$ in this film was $\sim 711 \mathrm{eV}$ and could be ascribed to both $\mathrm{Fe}(\mathrm{II})$ and $\mathrm{Fe}(\mathrm{III})$ states. $^{37}$

DFT calculations were carried out to investigate metal exchange observed experimentally by cyclic voltammetry and optical spectroscopy. Formation energies $(\Delta G)$ were calculated for each metal complex using the chemical stoichiometry shown in eqn (1)-(3). The $\Delta G$ values were compared between cations to give relative binding preferences (Fig. 6).

The calculations show a relative preference for $\mathrm{Zn}^{2+}$ $\left(-77 \mathrm{~kJ} \mathrm{~mol}^{-1}\right)$ and $\mathrm{Cu}^{2+}\left(-157 \mathrm{~kJ} \mathrm{~mol}^{-1}\right)$ complexes compared to the $\mathrm{Fe}^{2+}$ equivalent. This supports the experimentally observed exchange of $\mathrm{Fe}^{2+}$ to $\mathrm{Cu}^{2+}$ and $\mathrm{Zn}^{2+}$. The $\mathrm{Cu}^{2+}$ and $\mathrm{Fe}^{2+}$ results are consistent with binding constants for $\mathrm{Cu}^{2+}$ and $\mathrm{Fe}^{2+}$ with the nitrogen binding ligand 2,2-bipyridine. ${ }^{39-41}$ A difference in energy between the cations is likely due to $\mathrm{N}$-metal binding affinities and preferential binding geometries. The predicted $\mathrm{N}-$

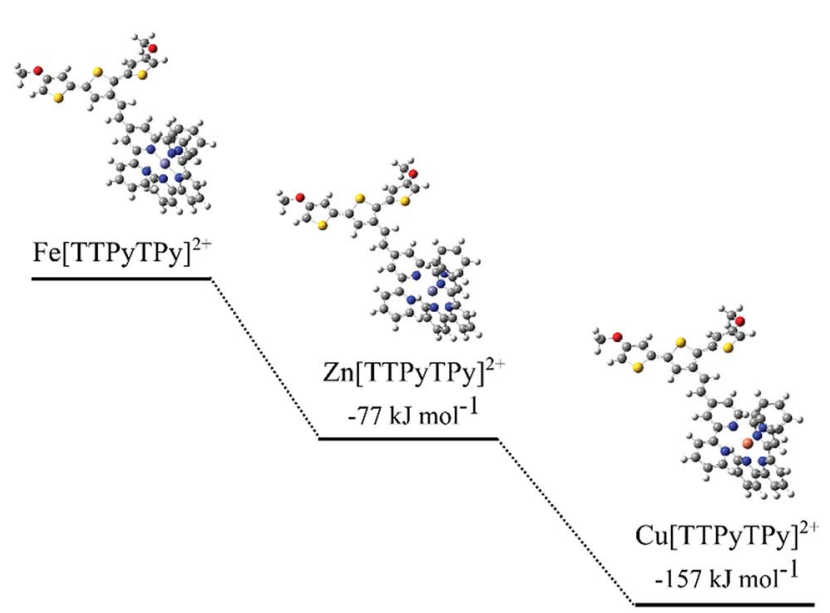

Fig. 6 Structural representations of modelled TTPy complexes and calculated $\Delta G$ values, relative to Fe[TTPyTPy $]^{2+}$.
Table 2 Average predicted bond lengths and ligand angles. Ligand angles were averaged between the $5,4^{\prime}$ and $5^{\prime \prime}$ carbons on both TTPy and TPy

\begin{tabular}{lll}
\hline & N-M bond length $/ \AA$ & $5,4^{\prime}, 5^{\prime \prime}$ bond angle/ \\
\hline Fe[TTPyTPy $]^{2+}$ & 2.00 & 82.9 \\
Zn[TTPyTPy] ${ }^{2+}$ & 2.22 & 88.0 \\
Cu[TTPyTPy $]^{2+}$ & 2.17 & 88.5
\end{tabular}

metal distances were explored in more detail in Table 2. Some differences in the geometries between the metal complexes were observed. For example, a 'clamped' geometry was predicted for Fe[TTPyTPy ${ }^{2+}$ with short N-Fe bonds $(2.00 \AA)$ and a distorted terpyridine structure $\left(5,4^{\prime}, 5^{\prime \prime}\right.$ bond angle of $82.9^{\circ}$ versus $93.4^{\circ}$ for bare terpyridine). $\mathrm{Zn}[\text { TTPyTPy }]^{2+}$ exhibits the longest $\mathrm{N}-\mathrm{M}$ bonds at $2.22 \AA$, while $2.17 \AA$ is predicted for $\mathrm{Cu}$ $[\text { TTPyTPy }]^{2+}$ with the least ligand angle deformation. These results suggest that $\mathrm{Cu}^{2+}$ binding results in the least distortion of the TPy residue and may be more highly conjugated within the terpy residue. In contrast, $\mathrm{Fe}^{2+}$ binding results in the largest distortion, which goes some way to support the experimental binding trends $\left(\mathrm{Cu}^{2+}>\mathrm{Zn}^{2+}>\mathrm{Fe}^{2+}\right)$.

In accordance with the results for $\mathrm{Cu}^{2+}$ shown above, a reduced $\mathrm{Cu}^{+}$cation was also considered. This is especially valid as $\mathrm{Cu}^{+}$is more stable than $\mathrm{Cu}^{2+}$ in acetonitrile and reduction of $\mathrm{Cu}^{2+}$ may occur. ${ }^{42} \mathrm{Cu}[\text { TTPyTPy }]^{+}$complex were predicted to be less favorable compared to $\mathrm{Cu}[\mathrm{TTPyTPy}]^{2+}$ (a difference of $+35 \mathrm{~kJ} \mathrm{~mol}^{-1}$ ). The energy difference is thought to be due to the well-established preferential geometries of $\mathrm{Cu}^{+}$ and $\mathrm{Cu}^{2+} \cdot \mathrm{Cu}^{+}$favors a tetrahedral arrangement and $\mathrm{Cu}^{2+}$ favors a trigonal bipyramidal arrangement. ${ }^{\mathbf{4 3 4}}$ The binding pockets of $\mathrm{TPy}_{2}$ and TPPyTPy are more trigonal bipyramidal like, hence are best suited to a $\mathrm{Cu}^{2+}$ cation. When considering $\mathrm{Zn}^{2+}$ and $\mathrm{Fe}^{2+}$ cations, $\mathrm{Cu}^{+}$would be preferred $\left(-45\right.$ and $-122 \mathrm{~kJ} \mathrm{~mol}^{-1}$ respectively).

\section{Conclusions}

The electropolymerization of a thin conducting polymeric film based on the repeating unit of $(E)-4^{\prime}-\left(2-\left(4,4^{\prime \prime}\right.\right.$-bisdecyloxy$2,2^{\prime}: 5^{\prime}, 2^{\prime \prime}$-terthiophen-3'-yl)ethenyl)-2,2' $: 6^{\prime}, 2^{\prime \prime}$-terpyridine (TTPy) was achieved by the application of cyclic voltammetry. Further on, the same polymeric material was found useful in tuning its electrochemical and optical properties by application of molecular architecture via surface and bulk modifications of the conducting film. The modification was done by different metal ions and their terpyridine complexes in various solvents (ACN and 1:1 ACN : $\mathrm{H}_{2} \mathrm{O}$ ). The electrochemical and optical properties of the conducting polymer films changed dramatically, namely films modified by $\mathrm{Fe}^{2+}$ and $\mathrm{Fe}^{2+}$-TPy resulted, in comparison to pure poly(TTPy), in an increased electrochemical capacitance, better conjugation of the conducting polymer system and higher absorptivity of the obtained film. Further exchange of the $\mathrm{Fe}^{2+}$ to $\mathrm{Zn}^{2+}$ and $\mathrm{Cu}^{2+}$ resulted in different electrochemical and optical properties of the obtained modifications. The electrochemical capacitance of the $\mathrm{Zn}^{2+}$ modified 
conducting polymer was maintained while optical properties of the film changed, while the electrical capacitance of the $\mathrm{Cu}^{2+}$ modified film significantly decreased indicating deterioration of the conducting polymer structure.

The surface and bulk modification of the polymeric film as demonstrated here may lead to the application of this methodology to obtain a material with tunable optical and electrochemical properties. Such modification may be obtained, in poly(TTPy), by application of different metal ions and various ligands equipped with terpyridine functionality as well as application of various solvents during the modification. Such materials may have application in changeable and switchable surfaces and intelligent sensors.

\section{Conflicts of interest}

There are no conflicts to declare.

\section{Acknowledgements}

Financial support to GL from the Åbo Akademi University's Research Foundation and FP7-PEOPLE-2010-IRSES, Materials and Advanced Sensor Knowledge Exchange Program (MASK) and Academy of Finland (project number 295019) is gratefully acknowledged. Aspects of this work carried out in the ARC Centre of Excellence for Electromaterials Science and Intelligent Polymer Research Institute at the University of Wollongong was supported by funding from the Australian Research Council (ARC) Centre of Excellence Scheme (Project Number CE 140100012) and the Australian National Fabrication Facility. KCG, GF and JEB thank the MacDiarmid Institute for support. JEB thanks the New Zealand eScience Infrastructure (NeSI) for computational time.

\section{Notes and references}

1 P. Manca, M. I. Pilo, G. Sanna, A. Zucca, G. Bergamini and P. Ceroni, Chem. Commun., 2011, 47, 3413-3415.

2 G. D. Storrier, S. B. Colbran and D. B. Hibbert, Inorg. Chim. Acta, 1995, 239, 1-4.

3 M. Wagner, G. Lisak, A. Ivaska and J. Bobacka, Sens. Actuators, B, 2013, 181, 694-701.

4 G. Lisak, K. Wagner, P. Wagner, J. E. Barnsley, K. C. Gordon, J. Bobacka, G. G. Wallace, A. Ivaska and D. L. Officer, Synth. Met., 2016, 219, 101-108.

5 P. C. Mondal, N. Kantor-Uriel, S. P. Mathew, F. Tassinari, C. Fontanesi and R. Naaman, Adv. Mater., 2015, 27, 19241927.

6 G. E. Collis, A. K. Burrell, S. M. Scott and D. L. Officer, J. Org. Chem., 2003, 68, 8974-8983.

7 J. S. Field, J. Haines, E. I. Lakoba and M. Hal Sosabowski, J. Chem. Soc., Perkin Trans. 1, 2001, 1, 3352-3360.

8 G. E. Collis, A. K. Burrell and D. L. Officer, Tetrahedron Lett., 2001, 42, 8733-8735.

9 U. S. Schubert and C. Eschbaumer, Angew. Chem., Int. Ed., 2002, 41, 2892-2926.
10 M. A. R Meier, B. G. G. Lohmeijer and U. S. Schubert, J. Mass Spectrom., 2003, 38, 510-516.

11 B.-S. Jeong, H.-Y. Choi, Y.-S. Kwak and E.-S. Lee, Bull. Korean Chem. Soc., 2011, 32, 3566-3570.

12 C. W. Machan, M. Adelhardt, A. A. Sarjeant, C. L. Stern, J. Sutter, K. Meyer and C. A. Mirkin, J. Am. Chem. Soc., 2012, 134, 16921-16924.

13 J.-C. Eloi, L. Chabanne, G. R. Whittell and I. Manners, Mater. Today, 2008, 4, 28-36.

14 R. Shunmugam, G. J. Gabriel, K. A. Aamer and G. N. Tew, Macromol. Rapid Commun., 2010, 31, 784-793.

15 D. F. Qiu, Q. Zhao, X. Bao, K. Liu, H. Wang, Y. Guo, L. Zhang, J. Zeng and H. Wang, Inorg. Chem. Commun., 2011, 14, 296299.

16 F. D. Jochum, J. Brassinne, C.-A. Fustin and J.-F. Gohy, Soft Matter, 2013, 9, 2314-2320.

17 R. Dong, Y. Zhou, X. Huang, X. Zhu, Y. Lu and J. Shen, Adv. Mater., 2015, 27, 498-526.

18 R. Sakamoto, K.-H. Wu, R. Matsuoka, H. Maeda and H. Nishihara, Chem. Soc. Rev., 2015, 44, 7698-7714.

19 C. Fan, C. Ye, X. Wang, Z. Chen, Y. Zhou, Z. Liang and X. Tao, Macromolecules, 2015, 48, 6465-6473.

20 D. K. Grant and D. L. Officer, Synth. Met., 2005, 154, 93-96. 21 M. Kimura, T. Horai, K. Hanabusa and H. Shirai, Adv. Mater., 1998, 10, 459-462.

22 A. Ciesielski, C.-A. Palma, M. Bonini and P. Samori, Adv. Mater., 2010, 22, 3506-3520.

23 G. E. Collis, W. M. Campbell, D. L. Officer and A. K. Burrell, Org. Biomol. Chem., 2005, 3, 2075-2084.

24 S. Gambhir, K. Wagner and D. L. Officer, Synth. Met., 2005, 154, 117-120.

25 D. K. Grant, K. W. Jolley, D. L. Officer, K. C. Gordon and T. M. Clarke, Org. Biomol. Chem., 2005, 3, 2008-2015.

26 K. Wagner, L. L. Crowe, P. Wagner, S. Gambhir, A. C. Partridge, J. C. Earles, T. M. Clarke, K. C. Gordon and D. L. Officer, Macromolecules, 2010, 43, 3817-3827.

27 K. Wagner, R. Byrne, M. Zanoni, S. Gambhir, L. Dennany, R. Breukers, M. Higgins, P. Wagner, D. Diamond, G. G. Wallace and D. L. Officer, J. Am. Chem. Soc., 2011, 133, 5453-5462.

28 R. Horvath and K. C. Gordon, Coord. Chem. Rev., 2010, 254, 2505-2518.

29 M. J. Frisch, et al., in Gaussian, Inc., Wallingford, 2009, p. 09. 30 A. Sezai Sarac, U. Evans, M. Serantoni, J. Clohessy and V. J. Cunnane, Surf. Coat. Technol., 2004, 182, 7-13.

31 D. Kim, J. Yoon, M. Won and Y. Shim, Electrochim. Acta, 2012, 67, 201-207.

32 R. Siebert, D. Akimov, M. Schmitt, A. Winter, U. S. Schubert, B. Dietzek and J. Popp, ChemPhysChem, 2009, 10, 910-919.

33 M. Jadamiec, M. Lapkowski, D. L. Officer, P. Wagner and K. C. Gordon, Int. J. Nanotechnol., 2009, 6, 344-354.

34 A. Milani, L. Brambilla, M. Del Zoppo and G. Zerbi, J. Phys. Chem. B, 2007, 111, 1271-1276.

35 H. Gato and E. Yashima, J. Am. Chem. Soc., 2002, 124, 79437949.

36 V. M. Donnelly and M. E. Gross, J. Vac. Sci. Technol. A, 1993, 11, 66-77. 
37 J. C. W. Folmer and F. Jellinek, J. Less-Common Met., 1980, 76, 153-162.

38 J. F. Moulder, W. F. Stickle, P. E. Sobol and K. D. Bomben, Handbook of X-ray Photoelectron Spectroscopy: A reference book of standard spectra for identification and interpretation of XPS data, Physical Electronics Division, Perkin-Elmer Corporation, 1992.

39 W. A. E. McBryde, Critical Evaluation of Equilibrium Constants in Solution: Stability Constants of Metal Complexes, Elsevier, 2013.
40 D. K. Hazra and S. C. Lahiri, Anal. Chim. Acta, 1975, 79, 335340.

41 F. Pantani, Ric. Sci., 1967, 37, 33.

42 B. Kratochvil, D. A. Zatko and R. Markuszewski, Anal. Chem., 1966, 38, 770-772.

43 M. G. Fraser, H. Salm, S. A. Cameron, J. E. Barnsley and K. C. Gordon, Polyhedron, 2013, 52, 623-633.

44 J. E. Barnsley, S. Ø. Scottwell, A. B. Elliott, K. C. Gordon and J. D. Crowley, Inorg. Chem., 2016, 55, 8184-8192. 